\title{
Analytical study of uneven distribution of seeds by a centrifugal distributor
}

\author{
Azamat E. Bogus ${ }^{1, *}$ \\ ${ }^{1}$ Chair of «Processes and machines in agribusiness» of the faculty of mechanization of Kuban State \\ Agrarian University named after I.T.Trubilin, 350044, Krasnodar, Russia
}

\begin{abstract}
The operating and geometric parameters of the seed movement in the centrifugal distributor of pneumatic grain seeders were studied on the basis of the equation of seed movement. The methodology of evaluation of the non-uniform distribution of seeds between the seed tubes is shown. The parameters of the centrifugal distributor that ensure minimal unevenness are determined.
\end{abstract}

\section{Relevance of the research}

In recent years, pneumatic grain seeders have become widely used allowing to increase the productivity of sowing units as well as to reduce operating costs [3, 5]. The main disadvantage of existing designs of pneumatic grain seeders is the uneven distribution of seeds between seed lines, which leads to overspending of seeds [4]. Kuban State Agrarian University has proposed a design of a centrifugal seed distributor [1], which allows reducing the unevenness of distribution along seed pipes. The design scheme of the distributor requires a theoretical justification of the design and operating parameters based on the equation of motion in the distributor.

\section{Investigation of uneven distribution of seeds by a centrifugal distributor}

The particle movement in the centrifugal seed distributor of a pneumatic seeder is described by the equation:

$$
h=\frac{g t^{2}}{2}-\frac{\frac{3 C_{c} u_{6}^{2} \rho_{b}}{8 R_{c} \rho_{c}} \sin \beta\left\{\frac{8 R_{c} \rho_{c} \omega^{2} R_{6} \cos \left(\varphi_{0}+\omega t\right)}{3 C_{c} u_{6}^{2} \rho_{6}}-\frac{8 R_{c} \rho_{c} R_{6} \omega^{2}}{3 C_{c} u_{6}^{2} \rho_{b} \cos \beta}\right\}}{\omega^{2}}+\frac{\omega^{2} R_{\sigma} \sin \beta \sin \left(\varphi_{0}+\omega t\right)}{\omega^{2}}
$$

where $C_{c}$ - coefficient of aerodynamic drag of a particle;

$$
\begin{aligned}
& \rho_{e}-\text { air density, } \mathrm{kg} / \mathrm{m}^{3} ; \\
& u_{e}-\text { air velocity, } \mathrm{m} / \mathrm{s} ; \\
& m_{c}-\text { weight of a single seed, } \mathrm{kg} ;
\end{aligned}
$$

\footnotetext{
*Corresponding author: azamat089@gmail.com
} 
$R_{c}$-radius of a single seed, $\mathrm{m}$;

$\rho_{c}$ - density of seeds, $\mathrm{kg} / \mathrm{m}^{3}$; deg;

$\alpha$ - angle between the speed direction of the point $A$ at the entrance to the distributor,

$\beta$ - angle between the direction of the absolute speed of the point $A$ and the plane and bottom of the distributor, deg;

$\varphi_{0}$ - initial angular coordinate of the seed at the entrance to the distributor chamber,

deg;

$R_{\sigma}$ - radius of the seed distributor

$u_{s}$ - air velocity, $\mathrm{m} / \mathrm{s}$;

$\omega$ - angular velocity of the seed, $\mathrm{s}^{-1}$;

$u$ - average air velocity, $\mathrm{m} / \mathrm{s}$;

$g$ - acceleration of free falling $\mathrm{m} / \mathrm{s}^{2}$;

$t$ - time, s.

Equation 1 describes the relationship between the geometric and operating parameters of the seeder, it is nonlinear and can be solved using a computer.

Ranges of variation of operating and geometric parameters of the centrifugal seed distributor were selected on the basis of a priori information (table 1).

Table 1. Ranges of variation of operating and geometric parameters of the centrifugal seeder.

\begin{tabular}{|l|c|c|c|c|c|}
\hline Parameter & \multicolumn{5}{|c|}{ Value } \\
\hline $\begin{array}{l}\text { Radius of the seeder } \\
R_{\sigma}, \mathrm{m}\end{array}$ & 0,15 & 0,20 & 0,25 & 0,3 & 0,35 \\
\hline $\begin{array}{l}\text { Velocity of seed } \\
\text { movement } u, \mathrm{~m} / \mathrm{s}\end{array}$ & 5 & 10 & 15 & 20 & 25 \\
\hline
\end{tabular}

To analyze the trajectories of seed movement, we placed them at the junction of the feed pipe and in the chambers of the centrifugal distributor (figure 1). To simplify the mathematical model, we assume that the seeds from all five points are immediately involved in a rotational movement along the walls of the distributor chamber, descending to the discharge pipes [2].

The initial angles of the seed's initial position at the point $1 \varphi_{1}$, are constant for the distributor of any radius of the distributor and are equal $\frac{\pi}{2}$, and the angles $\varphi_{2}, \varphi_{3,4,5}$ were calculated using formulas 2 and 3 according to figure 1 :

$$
\begin{gathered}
\varphi_{2}=\arcsin \left(\frac{R_{\sigma}-2 r}{R_{\sigma}}\right) \\
\varphi_{3,4,5}=\arcsin \left(\frac{R_{\sigma}-r}{R_{\sigma}}\right)
\end{gathered}
$$

The coordinates of points 1,2 , and 3 on the axis $Z$ at the initial time are equal to the height of the distributor $h$, the point 4 has a coordinate $h-r$, and the point 5 has a coordinate $h+r$. 


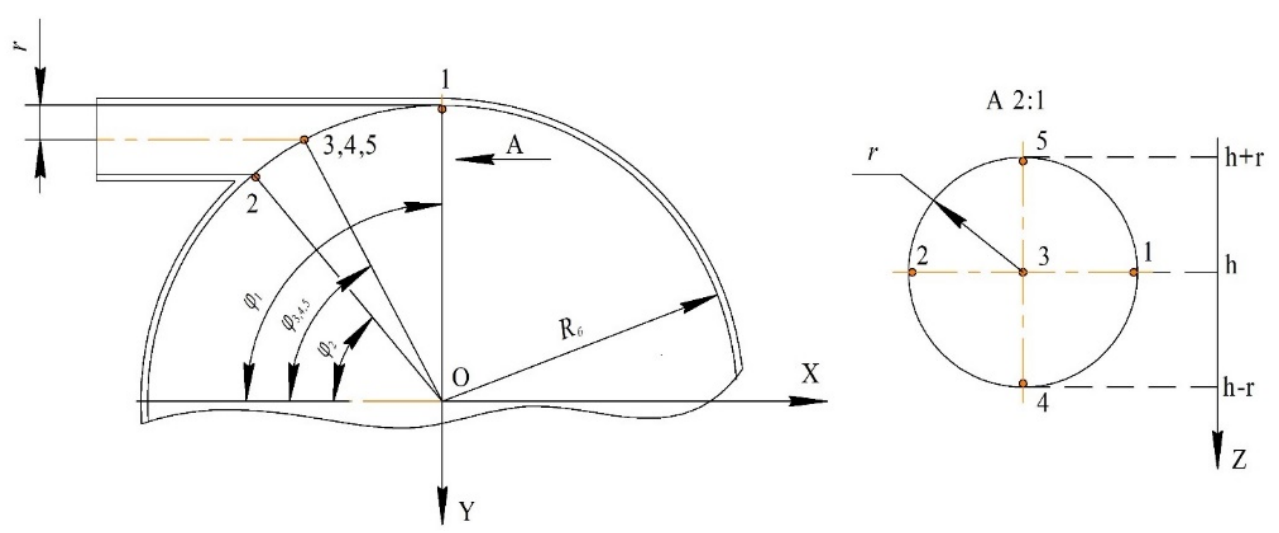

$\varphi_{1}, \varphi_{2}, \varphi_{3,4,5}$ - angular coordinate of seeds relative to the axes $X Y ; h$ - height of the distributor on the axis $Z, r$-radius of the inlet pipe; $R_{\sigma}$ - radius of the seed distributor

Fig. 1. Coordinates of analyzed seeds.

The values of the initial seed position angles at points 1, 2, 3, 4 and 5 for different radii of the centrifugal distributor are shown in table 2

Table 2. Values of the initial seed position angles.

\begin{tabular}{|l|l|l|l|l|l|}
\hline$R_{\sigma}, \mathrm{m}$ & 0,15 & 0,20 & 0,25 & 0,3 & 0,35 \\
\hline$\varphi_{1}, \mathrm{rad}$ & 1,570 & 1,570 & 1,570 & 1,570 & 1,570 \\
\hline$\varphi_{2}, \mathrm{rad}$ & 0,486 & 0,644 & 0,748 & 0,823 & 0,881 \\
\hline$\varphi_{3,4,5}, \mathrm{rad}$ & 0,823 & 0,927 & 0,997 & 1,048 & 1,088 \\
\hline
\end{tabular}

To estimate the uniformity of the distribution of seeds between the seed pipes at different regime and geometric parameters, we will substitute variable values in the transcendental equation 1 [2]. By setting the height of the distributor, we can determine the time $t$ in which each of the five particles will overcome the height $h$ from the inlet pipe to the bottom of the chamber connected with the outlet pipes, which will allow us to determine the final angle $\Theta$ at which the bottom will touch. The value of the angle $\Theta$ for all the points under study is determined by the formula:

$$
\Theta_{i}=\varphi_{i}+\omega t_{i}
$$

where $i$ - number of the point

The values of the angle $\Theta$ at different modes for the five studied are shown in table 3.

To determine the probability of uneven distribution of seeds, we determined the final scattering angle $\Delta \Theta_{\max }$ - the difference between the largest and smallest angular angles $\Theta$ [6]. In figures 2 and 3 there were shown the graphs of changes $\Delta \Theta_{\max }$ from the radius of the distributor $R_{\sigma}$ at different speeds of seed movement $u$, and from the speed of seed movement $u$ at different radii of the distributor $R_{\tilde{\sigma}}$. 
Table 3. Angle $\Theta$ at different regimes.

\begin{tabular}{|c|c|c|c|c|c|c|c|}
\hline$R_{\sigma}, \mathrm{m}$ & $u, \mathrm{~m} / \mathrm{s}$ & $\Theta_{1}$ & $\Theta_{2}$ & $\Theta_{3}$ & $\Theta_{4}$ & $\Theta_{5}$ & $\Delta \Theta_{\max }$ \\
\hline \multirow{6}{*}{0,15} & 5 & 291,51 & 229,34 & 252,95 & 240,06 & 266,24 & 62,16 \\
\hline & 10 & 133,01 & 76,57 & 93,02 & 70,10 & 121,66 & 62,91 \\
\hline & 15 & 8,89 & 321,73 & 348,89 & 318,81 & 14,67 & 340,0 \\
\hline & 20 & 303,98 & 241,82 & 184,75 & 89,64 & 337,56 & 247,91 \\
\hline & 25 & 347,91 & 285,74 & 314,62 & 104,57 & 21,48 & 326,42 \\
\hline & 30 & 16,59 & 314,43 & 357,80 & 104,55 & 42,49 & 341,2 \\
\hline \multirow{6}{*}{0,2} & 5 & 311,56 & 251,29 & 271,80 & 253,18 & 287,56 & 60,27 \\
\hline & 10 & 124,41 & 79,90 & 93,25 & 70,33 & 116,16 & 54,08 \\
\hline & 15 & 13,18 & 337,26 & 349,18 & 314,80 & 19,26 & 335,99 \\
\hline & 20 & 164,55 & 111,43 & 121,92 & 76,08 & 253,70 & 177,61 \\
\hline & 25 & 46,15 & 353,04 & 30,74 & 354,93 & 66,55 & 324,19 \\
\hline & 30 & 330,73 & 277,62 & 302,43 & 87,57 & 2,59 & 328,14 \\
\hline \multirow{6}{*}{0,25} & 5 & 284,35 & 237,19 & 247,16 & 232,84 & 262,92 & 51,5 \\
\hline & 10 & 173,12 & 103,04 & 128,77 & 77,20 & 168,88 & 95,9 \\
\hline & 15 & 8,89 & 321,73 & 348,89 & 318,81 & 14,67 & 340,0 \\
\hline & 20 & 77,58 & 30,49 & 58,50 & 31,00 & 86,01 & 55,51 \\
\hline & 25 & 130,17 & 83,02 & 108,74 & 74,36 & 263,44 & 189,07 \\
\hline & 30 & 347,71 & 300,56 & 328,57 & 108,56 & 9,83 & 337,88 \\
\hline \multirow{6}{*}{0,3} & 5 & 95,73 & 52,93 & 71,27 & 62,84 & 79,17 & 42,79 \\
\hline & 10 & 103,34 & 58,65 & 79,16 & 63,92 & 92,57 & 44,69 \\
\hline & 15 & 104,36 & 61,50 & 82,99 & 65,80 & 105,91 & 44,4 \\
\hline & 20 & 109,11 & 66,31 & 86,82 & 63,90 & 117,41 & 53,15 \\
\hline & 25 & 109,15 & 66,30 & 93,51 & 64,86 & 126,97 & 62,10 \\
\hline & 30 & 112,98 & 70,12 & 100,20 & 65,83 & 277,82 & 211,99 \\
\hline \multirow{6}{*}{0,35} & 5 & 50,07 & 10,53 & 27,26 & 20,73 & 34,19 & 39,53 \\
\hline & 10 & 21,52 & 16,42 & 2,04 & 17,97 & 13,50 & 19,48 \\
\hline & 15 & 359,91 & 321,81 & 341,23 & 319,63 & 358,94 & 40,27 \\
\hline & 20 & 339,27 & 299,74 & 260,84 & 272,29 & 344,32 & 83,47 \\
\hline & 25 & 116,94 & 77,41 & 113,79 & 84,34 & 200,60 & 123,18 \\
\hline & 30 & 116,94 & 77,41 & 113,79 & 83,89 & 180,77 & 103,36 \\
\hline
\end{tabular}




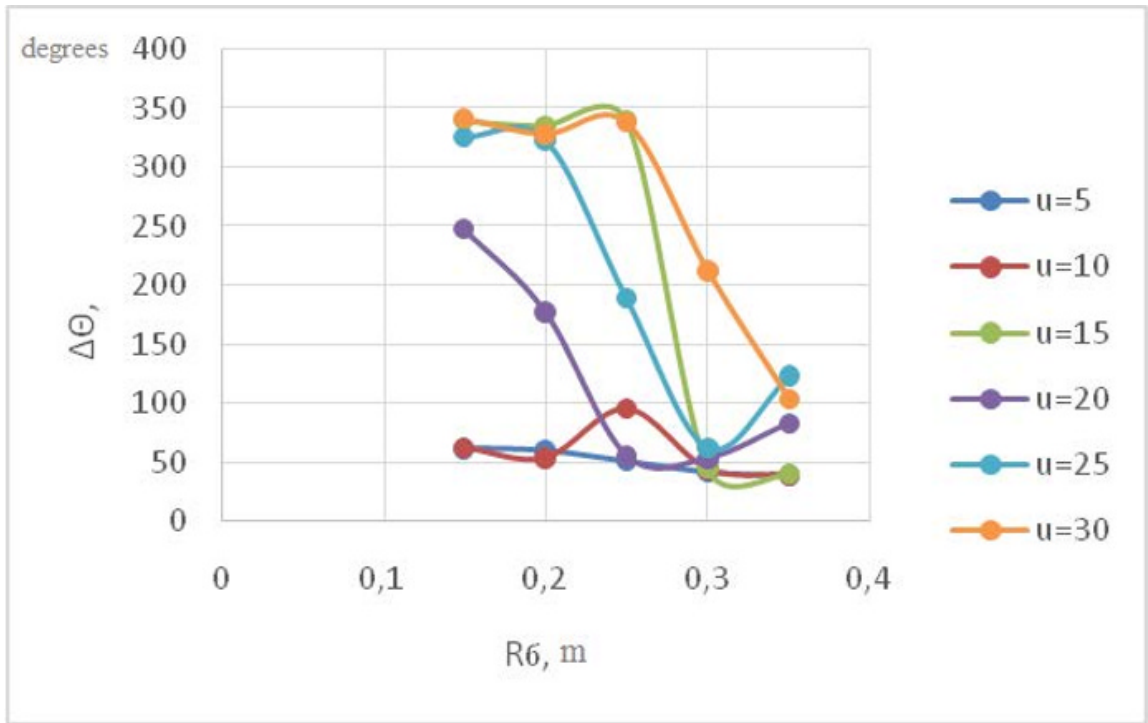

Fig. 2. Graph of the change $\Delta \Theta_{\max }$ from $R_{\sigma}$ at $u=5 \ldots 30 \mathrm{~m} / \mathrm{s}$

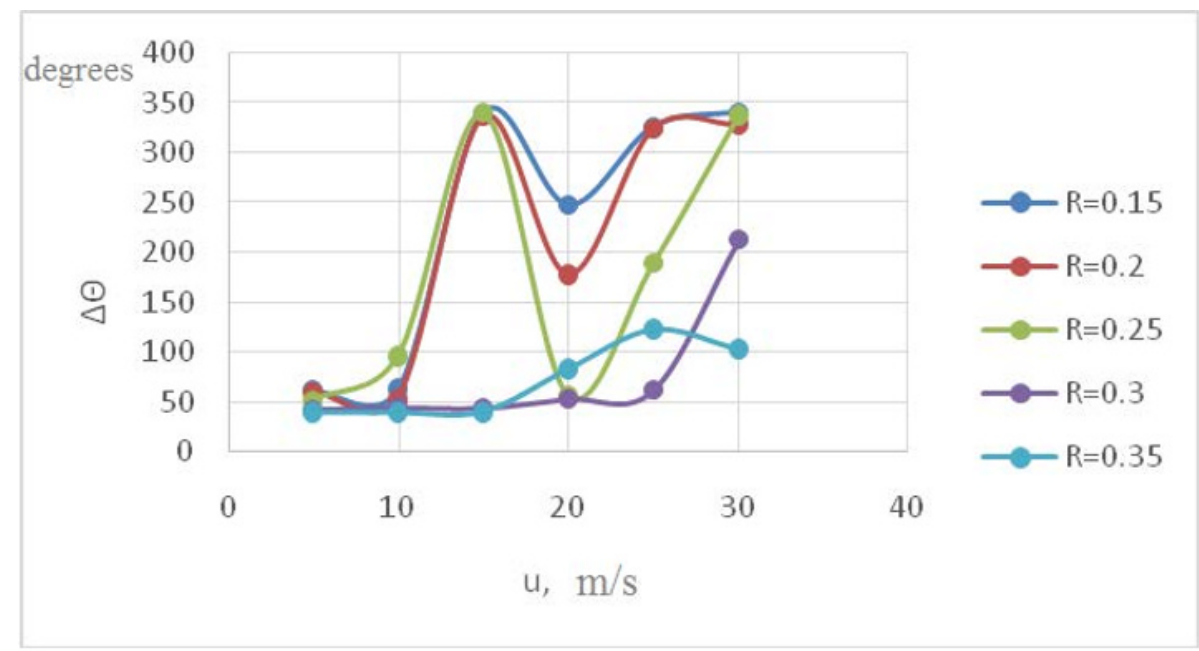

Fig. 3. Graphs of the change $\Delta \Theta_{\max }$ from $u$ at $R_{\sigma}=0,15 \ldots 0,35 \mathrm{~m}$

The design of the centrifugal seed distributor (figure 1) contains a bottom that is a ring with 28 holes. We calculate the probability $Q(A)$ that the scattering angle $\Delta \Theta_{\max }$ will evenly distribute the seeds that entered the chamber through a single inlet pipe along the ring by the expression:

$$
Q(A)=\frac{\Delta \Theta_{\max }}{2 \pi}
$$

For ease of analysis, let's go to the probability of uneven distribution $P(A)$ which is determined by the formula:

$$
P(A)=1-Q(A)
$$


From the diagrams of the dispersion angle dependence, it can be concluded that under certain modes and parameters of the distribution system, part of the seed flow from the two pipes may not intersect, i.e. be independent, and others may intersect, i.e. simultaneously affect the amount of uneven distribution [7,8]. Accordingly, for the case of non-intersecting flows, the total unevenness of the seed distribution will be determined by the sum of two irregularities, and for the case of intersection, it will be determined by the joint probability of unevenness of the distribution of two seed flows.

Since two inlet pipes are involved in the distribution process, it is not necessary to add up the probabilities of uneven distribution between the first pipe $P(A)$ and the second pipe $P(B)$. If the probability $P(A) \triangleleft 0,5$ is the sum of two probabilities calculated using the formula:

$$
P(A B)=P(A) P(B)
$$

In the case where the probability $P(A) \triangleright 0,5$ is the sum of two probabilities defined by the expression:

$$
P(A B)=1-Q(A)-Q(B)
$$

Table 4 shows the probability $P(A B)$ of uneven distribution of seeds by a centrifugal distributor at different operating and geometric parameters [9]. Graphs of the uneven distribution of seeds by a centrifugal distributor from the radius of the distributor at different speeds are presented in figure 4. Graphs of the uneven distribution of the speed of seed movement at different radii of the distributor are shown in figure 5.

Table 4. Probabilities $P(A B)$ of uneven seed distribution.

\begin{tabular}{|c|c|c|c|c|c|}
\hline \multirow{2}{*}{$\begin{array}{c}R_{\sigma}, \mathrm{m} \\
\mathrm{u}, \mathrm{m} / \mathrm{s}\end{array}$} & \multicolumn{5}{|c|}{$P(A B), \%$} \\
\hline & 0,15 & 0,2 & 0,25 & 0,3 & 0,35 \\
\hline 5 & 65,40 & 66,50 & 71,00 & 76,20 & 78,0 \\
\hline 10 & 65,00 & 84,90 & 46,00 & 75,10 & 89,1 \\
\hline 15 & 0,30 & 6,60 & 0,30 & 75,30 & 77,0 \\
\hline 20 & 9,00 & 1,30 & 69,10 & 70,20 & 53,60 \\
\hline 25 & 0,80 & 0,90 & 22,50 & 65,40 & 31,0 \\
\hline 30 & 0,27 & 0,78 & 0,37 & 16,90 & 42,0 \\
\hline
\end{tabular}

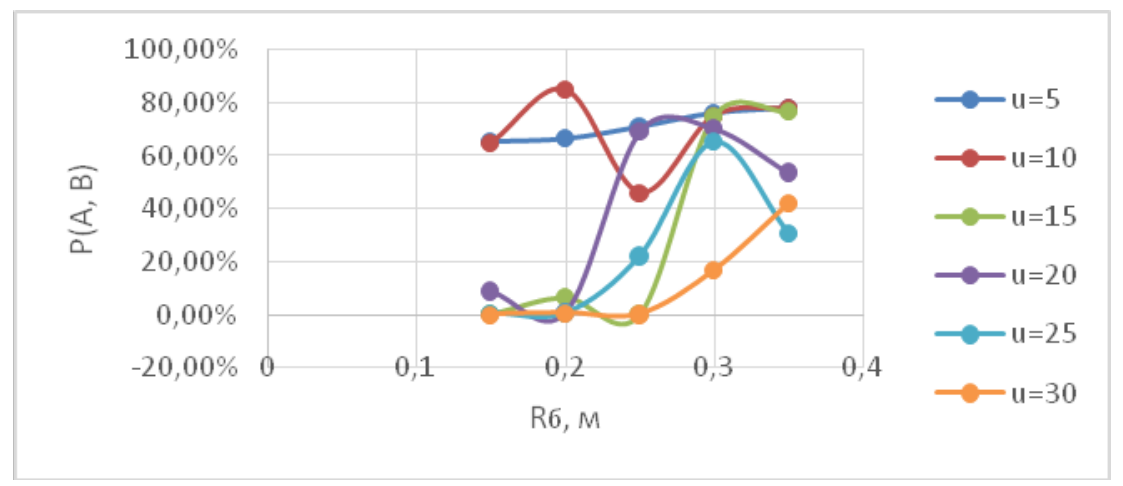

Fig. 4. Graphs of change $P(A, B)$ from the radius of the distributor $R_{\sigma}$ at different velocities $u$ 


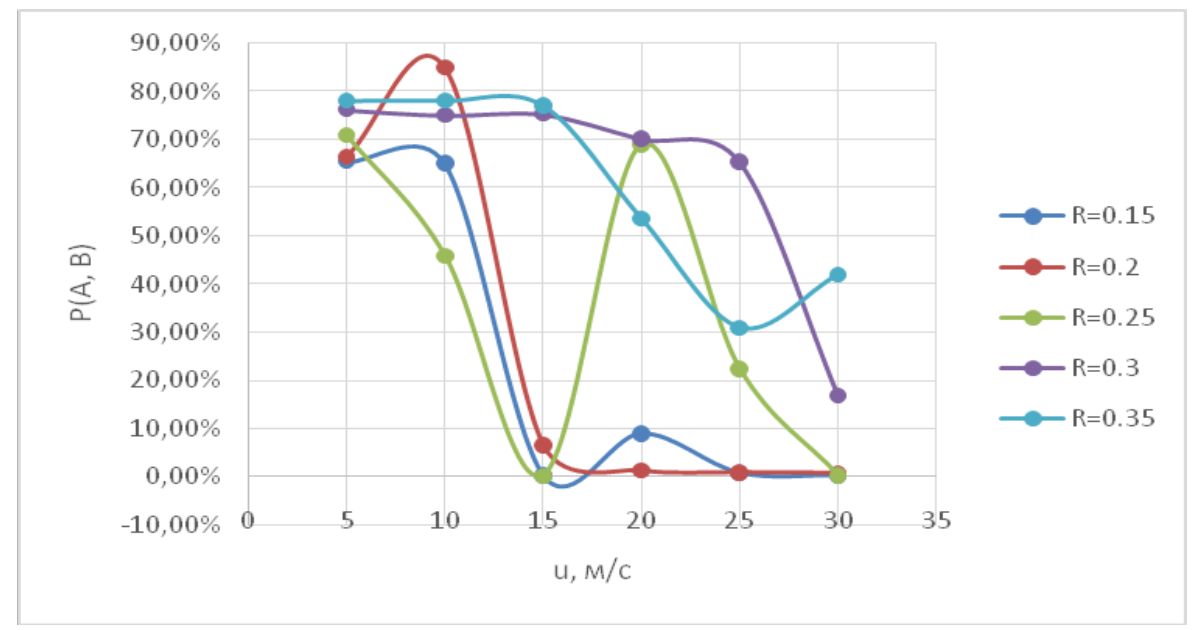

Fig. 5. Graphs of changes $P(A, B)$ from the velocity of seed movement at the radii of the distributor $R_{\sigma}$

From the graphs in figures 4 and 5 it can be seen that the minimum unevenness of seed distribution by the centrifugal seeder is achieved when the radius of the distributor $R_{\sigma}$ is equal to 0.15 and $0.2 \mathrm{~m}$ at seed movement speeds from 15 to $30 \mathrm{~m} / \mathrm{s}$. However, the most effective is a distributor with a radius of $0.2 \mathrm{~m}$ and a seed velocity of $15 \mathrm{~m} / \mathrm{s}$.

\section{Conclusions}

An analytical study of the uneven seed distribution by a centrifugal distributor allowed us to determine the operating and design parameters of the centrifugal distributor: the radius $R_{\sigma}=0,2 \mathrm{~m}$, the speed of movement of seeds $u=15 \mathrm{~m} / \mathrm{s}$ at which a minimum unevenness of $3 \%$ between the seed pipes is provided.

\section{References}

1. Pat. 2448444 Russian Federation, MPK A01C15/04. Pneumatic seeder with a central metering system [Text] / E. I. Trubilin, A. V. Khokhlov, A. A. Khokhlov, A. E. Bogus, V. V. Kutseev; applicant and patent holder, Federal State Budgetary Educational Institution of Higher Education "Kuban State Agrarian University named after I. T. Trubilin". - № 2010133832/13; claimed 12.08.2010; publ. 27.04.2012, Bul. № 12. - 13 p.: ill.

2. Rotary disk working bodies - as a basic element in combined aggregates for soil treatment and seeding / E. I. Trubilin, K. A. Sokht, V. I. Konovalov, O. V. Danyukova // Polythematic network electronic scientific journal of Kuban State Agrarian University Krasnodar.: Publ. house "KubSAU", 2013, \#. 91, Pp. 465-497.

3. Rybalkin P. N. New adaptive energy and soil-saving technologies of winter wheat and corn cultivation in Krasnodar Territory [Monograph] / Rybalkin P. N. [et al.]. Krasnodar: Russian Academy of agricultural Sciences, Krasnodar scientific research Institute of agriculture named after P. p. Lukyanenko, LLC "Enlightenment-Yug", 2002, $103 \mathrm{p}$.

4. Rybalkin P. N. Agrotechnical requirements for basic technological operations in adaptive technologies of cultivation of winter grains and corn and new technical units for their 
implementation in Krasnodar Territory [Recommendations] / Rybalkin P. N. [et al.]. Krasnodar: Department of Agriculture and Food of Krasnodar Territory, Russian Academy of Agricultural Sciences, Krasnodar Research Institute of Agriculture named after P. P. Lukyanenko, Publ. house LLC "Agroprompoligrafist", 2001. - 144 p.

5. Agricultural machines (equipment, operation and basic adjustments): manual / V. A. Romanenko [et al.]. - Krasnodar: KubSAU, 2014. - 232 p.

6. Statistical methods of researches of processes and machines in agribusiness [Text] / K.A. Sokht, E. I. Trubilin, V. I. Konovalov / Krasnodar: KubSAU, 2016. - 217 p.

7. Belousov S.V., Saprykin E.A., Karmazin I.S., Explanation of the angle of sharpening of a plough cutting working body, E3S Web of Conferences 2019. C. 00025.

8. Belousov S.V., Pomelyayko S.A., Novikov V.V., Design of the universal agricultural working body and study of its parameters, MATEC Web of Conferences 2018. C. 05006.

9. Belousov S.V., Gusak E.S., Theoretical justification of fruit separation process by a planetary fruit separator, E3S Web of Conferences 2019. C. 00056. 